\title{
Delphi based Auto Narrator for Web and Mobile Traffic Analysis
}

\author{
Paramjot Kaur Sarao \\ BBSB Engineering College \\ Fatehgarh Sahib \\ Punjab, INDIA
}

\author{
Puneet Mittal \\ BBSB Engineering College \\ Fatehgarh Sahib \\ Punjab, INDIA
}

\author{
Gursharan Singh Dhanjal \\ BBSB Engineering College \\ Fatehgarh Sahib \\ Punjab, INDIA
}

\begin{abstract}
Numbers do not make much sense while doing analysis Unless they are supported with narratives, remarks, Interpretations, deductions and explanations of these numbers and figures. This is skilled task that can be accomplished by experts. In this research work, the authors have built an automatic data analysis narrator of web/mobile traffic data. The method employed is Delphi to identify requirements of customers for data analysis report's ordering and using descriptive statistical analytical methods to build narration of web/mobile traffic. The reports are validated against the ground truth. The results show high level of acceptability of reports by users due to high recall and precision values.
\end{abstract}

\section{General Terms}

Narratives, Narration Generation, Analytics.

\section{Keywords}

Automatic Narration Generation; Delphi technique; Mobile analytics; Natural Language Generation; Quality Function Deployment; Web analytics.

\section{INTRODUCTION}

Narrative science systems can be considered as 'Robots' that know the 'art of story-telling' using mathematical algorithms.
These systems, try to treat 'objects' as entities just like humans with the capabilities directly proportional to the size of database, speed of retrieving relevant information and presentation of format quality. Examples of such commercial systems are Quill and Quill-Engage [1] by Narrative Science and Adobe Analytics [2] by Adobe. They are widely used in the various fields i.e. Research, News report-writings, Web report analysis, journalism, corporate communication, financial services etc. to enhance business value.

Most of the narrative science systems are based on the narrative paradigm theory [3], that states that a story is a series of actions/events that have beginning, middle points and end points, and have some meaningful format and ordering. However, the design and implementation of such narrative science systems are confronted with many challenges as illustrated below as per their stage of the design.

\subsection{Phases of building Narrative Science Systems}

The design of the Narrative science systems includes the following phases as discussed in "Table 1":

Table 1. Phases of Narrative Science systems with design issues

\begin{tabular}{|c|c|c|}
\hline Phase & Description & Issues \\
\hline Data Collection sets & $\begin{array}{l}\text { In this phase a large amount of data is collected, } \\
\text { stored and indexed for the future use. }\end{array}$ & $\begin{array}{l}\text { The collected data is domain specific and there is } \\
\text { greater need of the domain specific databases to store } \\
\text { that data. } \\
\text { There is a problem in calculating the complexity of the } \\
\text { huge data. }\end{array}$ \\
\hline $\begin{array}{l}\text { Identification of } \\
\text { story facts and } \\
\text { figures }\end{array}$ & $\begin{array}{l}\text { The collected data is analysed to identify the } \\
\text { story plots, format order, structure and } \\
\text { templates. }\end{array}$ & $\begin{array}{l}\text { - Knowledge of various data mining techniques is } \\
\text { required. } \\
\text { - The expectancy level of the users must be predefined as } \\
\text { the facts are to be identified according to the user's } \\
\text { need. }\end{array}$ \\
\hline $\begin{array}{l}\text { Mathematical rules } \\
\text { of inference and } \\
\text { deduction }\end{array}$ & $\begin{array}{l}\text { Different Artificial intelligence, reasoning or } \\
\text { Data mining techniques are applied to extract } \\
\text { the meaningful data. }\end{array}$ & $\begin{array}{l}\text { - Require large effort to build predefined rules to identify } \\
\text { the facts that may be necessary. } \\
\text { - There is a need of predefined semantic representation of } \\
\text { the data. } \\
\text { - It is not possible to find/discover the rules hidden in the } \\
\text { data without a sophisticated algorithm. }\end{array}$ \\
\hline $\begin{array}{l}\text { Pre-stored Narrative } \\
\text { sets }\end{array}$ & $\begin{array}{l}\text { Some narrative sets have been extracted and } \\
\text { stored for the narrative content mapping. }\end{array}$ & $\begin{array}{l}\text { - They are static in output. } \\
\text { - It may possible that meaningful information may not be } \\
\text { contained in the pre-stored narrative content. }\end{array}$ \\
\hline $\begin{array}{l}\text { Narrative content } \\
\text { semantic mapping }\end{array}$ & $\begin{array}{l}\text { The pre-stored narrative content is turned into } \\
\text { the narrative content with respect to the } \\
\text { mathematical rules and their semantics. }\end{array}$ & $\begin{array}{l}\text { - Most of the narrative science systems work on limited } \\
\text { aspects of the narrative structure like narration length, } \\
\text { angels etc. } \\
\text { - Knowledge of machine learning and artificial } \\
\text { intelligence algorithms is essential. }\end{array}$ \\
\hline
\end{tabular}




\begin{tabular}{|l|l|l|}
\hline & & $\begin{array}{l}\text { - It is difficult to measure the user expectancy level by } \\
\text { the generated narratives. } \\
\text { The story may be generated by setting different } \\
\text { parameters under simulated environment. }\end{array}$ \\
\hline $\begin{array}{l}\text { Quality Evaluation } \\
\text { and scoring }\end{array}$ & $\begin{array}{l}\text { The quality of the generated narrative is } \\
\text { evaluated with respect to the mapping and } \\
\text { insights expected from the narration in this } \\
\text { phase and scores have been given by the } \\
\text { experts. }\end{array}$ & $\begin{array}{l}\text { - As the story is object based, it may lack in creativity. } \\
\text { - It is not possible to do quality truth validation purely. } \\
\text { - The generated narrative may lack in expertise } \\
\text { agreement. }\end{array}$ \\
\hline $\begin{array}{l}\text { Delivery of auto } \\
\text { generated narration }\end{array}$ & $\begin{array}{l}\text { After mapping and quality evaluation, the } \\
\text { automatically generated narration is finally } \\
\text { delivered to the users. }\end{array}$ & $\begin{array}{l}\text { - The delivered insights may not satisfy users. } \\
\text { - The delivered insight is template based in nature. }\end{array}$ \\
\hline Feedback & $\begin{array}{l}\text { After delivering the output to the users, the } \\
\text { feedback is taken to measure the user's degree } \\
\text { of satisfaction and improve the system. }\end{array}$ & $\begin{array}{l}\text { - Objectives for taking feedback must be cleared prior to } \\
\text { taking feedback. }\end{array}$ \\
\hline Need of an organized customer feedback system.
\end{tabular}

\section{RELATED WORK}

The integration of the prototype of the thematic model in the existing narrative generation had been proposed by Charlie Hargood et al. in [4]. The thematic model described how the features of the narratives reports or essays are themselves decorative designs that further in turn specify the themes. The integration of themes can nourish the stories that were lacking in variety and having no interesting features. The authors also described the challenges of integration of model with various narration generation approaches like author centric, character centric or compromise of both. The paper suggested that the integration had been done in such a manner that there was no damage to the narrative content. The stories that were generally tamed or dull would be of high quality and interest after the inclusion of themes. The quality of theme was measured by giving score using the algorithm on the basis of two metrics i.e. component coverage - was used for measuring how strong is the relationship between a feature and a desired theme, and thematic coverage - was used to find themes for which a feature has a relationship. It was observed that integration of themes seems more possible and can be thematically scored at the plotting level.

In [5] the narratives in the computer based simulation world have been modeled by R. Michael Young. According to him, the narration content was composed of story and discourse elements. The story elements included plot and characters and discourse elements such as actions, were defined in the form of plan based model. Narrative plans were generated by using the Decomposition Partial order Causal Link planner. The Mimesis system is a system used to develop interactive computer based simulated applications. This system generated plans (based on plan based model) for managing the different characters comprising the narratives and the different media resources that were narrating the narratives. It was also tried to manage interactivity within the story. The author had tried to manage interactivity in the narratives on the basis of controlling the characters operating within the narration and coherence. Coherence means establishing the relationship between the events, actions and story-telling. Control means the power of performer to influence the audiences by his/her character, the environment in which he/she is performing. Narrative mediation is the technique used in the paper for the allowed degree of control and coherence that a story can carry out. The suspense level was also determined using refinement planning algorithm- represented plans and searches in the form of directed graph. The narrative in XML format was generated in the virtual world with the integration of story and discourse plans to enhance the performance of the narrative generation system.

The implementation of the Natural Language Generation system named AUTHOR in STORYBOOK, for building the story with multi-paragraph text had been modeled in [6] by Charles B. Callaway et al. STORYBOOK was the full-fledged narrative prose generation system based on planning done by the narrative planner that incorporated syntactic, semantic and orthographic features. This architecture tried to remove the gaps between the story generators and the Natural Language Generation systems by focusing on the features of the prose generation. The thematic roles were also assigned to the concepts to increase the quality of the narratives. The quality of the narrative was also evaluated by narrative planner capable of generating stories in predefined specifications.

There was a discussion regarding the existing computational models that addressed the representation of the various aspects including discourse sequence, simulation, intention, theme, emotion, structure and authors that were mandatory for the narration generation in [7] by Pablo Gervas et al. The authors also discussed how humans faced the shifting between the views defined by the characters and the structure in the narrative research. The basic issues like multiple aspects representation by existing systems, finding the nature of the narratives by analyzing the aspects, relationship between them etc. were also discussed by the authors. The relationship between the aspects and the different data structures with respect to the representation of the narrative was also studied in the paper.

An experiment had been performed by Takashi Ogata et al. taking the focal point/view point and duration aspect of narratives into account in [8]. The authors performed this experiment for the aspects generally of the sports soccer game by using the Allegro Common Lisp- the most powerful object oriented system that transformed conceptual data which expressed the narration based on the different specified perspectives. The Authors took Genette's discourse theory as a base for the generation and evaluation of the narratives. The authors also tried to integrate the view point and duration aspects of the narratives.

A model called three-fold that showed a grouping of aspects including a group of players, rules for playing the games, scenarios of game playing etc. into logical categories include drama, game and simulation, of the story construction in the context of virtual world games had been presented by Crag A. Lindley in [9]. Drama dealt with game actions and game dealt 
with the style of gaming. The games were played at different levels that were based on simulation. The narration generation in the field of simulated computer games was based on interactivity involved at the different levels of games using the predefined time structures. The time structures were also based on time scales. The story generation in this paper was for computer based role playing games. The three-act restoration structure was used in this model for framing narratives in a recursive manner. The three act story restoration structure divided the narratives into three parts called setup, confrontation and resolution. Setup means defining characters and their relationships; confrontation means defining the situation in the games where players compete to win the game and resolution dealt with the climax of the story. The author suggested that an object oriented paradigm was best suited for constructing narrative structures as the narrative characters involved different emotions and relationships in a virtual life that were complex and of interest.

\section{SCOPE OF IMPROVEMENT}

It is clear from the above study that most of the data is generic in nature and few systems were working in the gaming domain. It has also been observed that few research papers have a quality check for the narration generated. The research papers referred for the automatic narration generation have ignored the quality validation and subject agreement by the experts. Most of the narration generation systems are evaluated with very few aspects and fewer of these systems give the ground quality truth validation of the narration's output. Many computational models have been developed to generate the narration interactively and to behave in the dynamic environment. The Natural Language generation systems have been evaluated on the basis of their content and performance [10]. In some of the cases, the objective evaluation is followed by subjective evaluation but none of the systems have an intervention of humans to decide narrative structure/plot/format of the narration during narration generation which leads to dull stories. The automatically generated story can be made like human crafted story by introducing the expertise evaluation parallel to the story generation algorithms, so that, there will be a no need to compromise with the discourse and quality of the narrative structure.

\section{PROPOSED AUTOMATIC NARRATION GENERATION SYSTEM}

After conducting systematic literature survey and narrowing down the scope of work based on gaps identified, the following system is proposed that overcomes the limitations of the previous works.

\subsection{Architecture of Proposed Automatic Narration Generation System}

The following "Fig 1" shows the basic architecture of the proposed automatic narration generation system. The System has seven main modules.

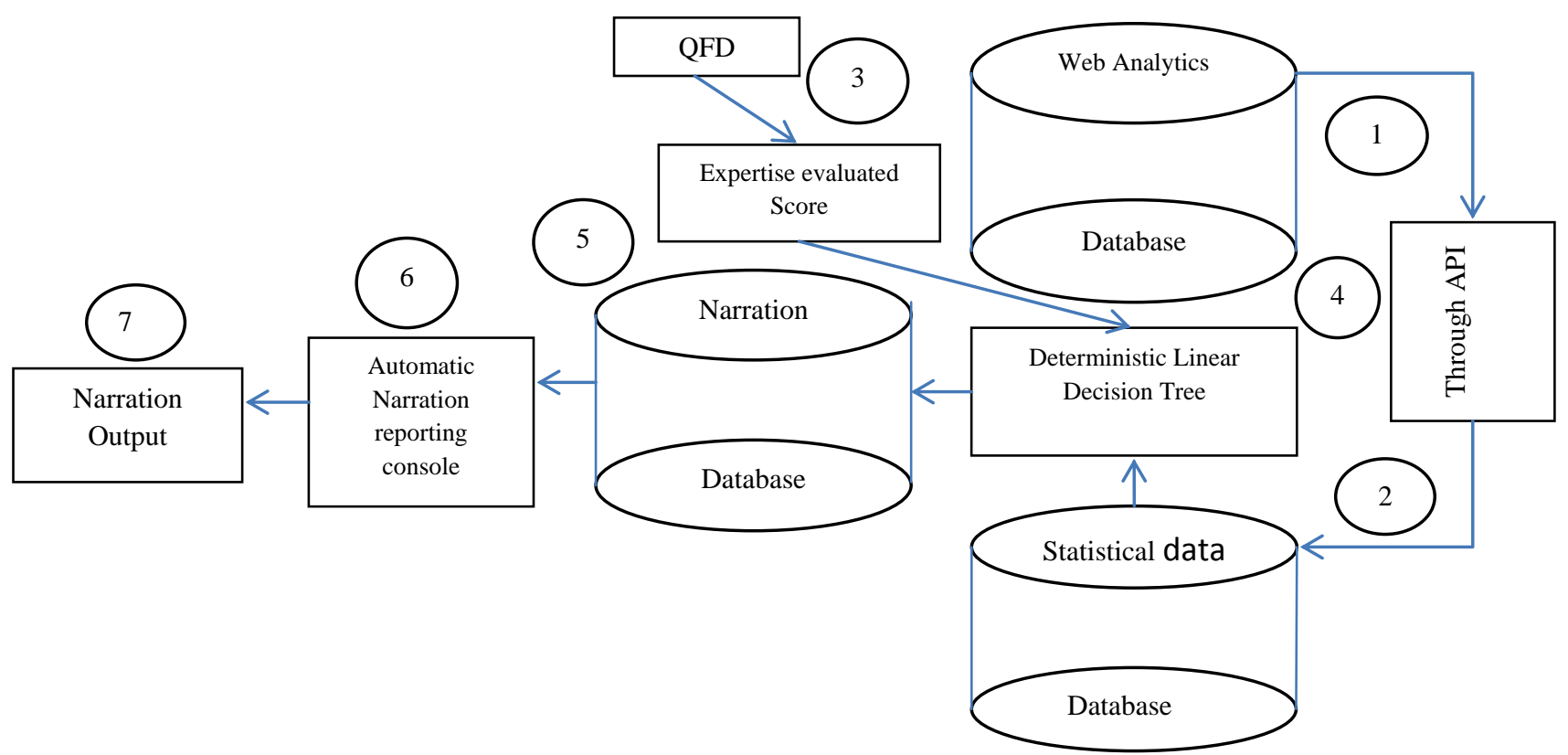

Fig 1: Architecture of Proposed Automatic Narration Generation System

Step 1: The first module consists of datasets from Web Analytics Domain specific event descriptors, collected through Google analytics [11] service for the website(s) under observation named www.punjabupdate.com and www.ijorcs.org.

Step 2: The second component consists of statistical data collected from the website(s) under observation using Google Analytics reporting application programming interfaces [12] and stored in the relational database i.e. My SQL-5.6. The statistical data is then collaborated with a particular set of event(s) format or plot. From this format the automatic generation algorithm picks its structure to output the narration.

Step 3: Next, the performance parameters of the website(s) are incorporated in the form of questions [13] in the questionnaire. The parameters are rated on the different scales [14] and the responses [15] of different raters for the questions are evaluated by using Delphi technique [16] and the degree of agreement between raters is measured by using Fleiss' kappa formula [17]. The degree of agreement between the raters on the questions is defined as, 
$K=\frac{\bar{P}-\bar{P}_{e}}{1-\bar{P}_{e}}$

Where, $K$ is agreement between raters,

$\bar{P}$, is the observed agreement,

$\overline{P_{e}}$, is the expected agreement.

Let $N=$ the total number of questions,

Let $n=$ the total number of raters assigned,

And let $k=$ the total number of scale rating values for which assignments are to be made. The questions are indexed by $i=1, \ldots, N(N=93)$ and the scale rating values are indexed by $j=1, \ldots, k(k=5$ as 5 scale rating is used).

Before calculating the value of $K$, first calculate $P_{j}$, the proportion of all assignments which were to $j^{\text {th }}$ different scale rating values for the responses collected through the questionnaire survey.

$p_{j}=\frac{1}{N n} \sum_{i=1}^{N} n_{i j}$

Where, $n_{i j}$ represents the number of raters who assigns $i^{\text {th }}$ question to the $j^{\text {th }}$ scale rating value.

Next is to calculate $P_{i}$, the extent at which raters agree for $i^{t h}$ question.

$P_{i}=\frac{1}{n(n-1)}\left[\left(\sum_{j=1}^{k} n_{i j}^{2}\right)-(n)\right]$

Then calculate $\bar{P}$ (mean of the $P_{i}$ 's and the observed agreement) and $\overline{P_{e}}$ (the expected agreement) to finally calculate the degree of agreement.

$\bar{P}=\frac{1}{N} \sum_{i=1}^{N} P_{i}$

And,

$\overline{P_{e}}=\sum_{j=1}^{k} p_{j}^{2}$

The outcome of the questionnaire on 45 raters shows the value $K=0.6210$, means 45 raters have the substantial agreement between them for 93 questions rated on the scale of 5 in the questionnaire using Delphi technique.

Also, the Quality function deployment (QFD) [18] is designed for the website(s) under observation to find out which technical design attribute may contribute maximum to improve the quality of the website. The QFD for website(s) under observation is designed using following steps:

Step 3.1: After collecting the responses for calculating the degree of agreement, next is to calculate the ratio of the extent to which the raters agree to the proportion of all assignments to the scale rating value [19], which is calculated by dividing the value of each $P_{i}$ of Fleiss' kappa formula for each question by the each value $p_{j}$ of the Fleiss' kappa formula and is recorded to decide the relevance of all the questions in next step.

Step 3.2: After calculating the ratio, the cut-off values(cutoff) for the ratio in different columns are calculated for the checking questions relevancy by the following formula: cutoff $=\mid$ Average $|+|$ Max.standard deviation $\mid$

The following "Table 2" represents the different calculated cut-off values for the ratio values calculated in the previous step.

Table 2. Different Cut-off values

\begin{tabular}{|c|c|c|}
\hline S no. & Column name & Cut-off value \\
\hline 1 & $P_{i} p_{j 1}$ & 111 \\
\hline 2 & $P_{i} p_{j 2}$ & 8.0 \\
\hline 3 & $P_{i} p_{j 3}$ & 2.2 \\
\hline 4 & $P_{i} p_{j 4}$ & 3.0 \\
\hline 5 & $P_{i} p_{j 5}$ & 3.0 \\
\hline
\end{tabular}

Step 3.3: The Questions whose ratio value in each column lies below and are in the range of all calculated cut-off values or below the minimum of 3 different cut-off values for each column are considered. The questions with ratio value which lie above all the calculated cut-off values or above maximum 4 different calculated cut-off values are not considered. As higher the value of the ratio, lesser is the relevance of that question. After checking the relevance of each question, it is observed that 79 no. of questions [20] are recorded as relevant.

Step 3.4: Finally, the precedence/order/priority value $\left(P_{o}\right)$, for the 79 relevant questions is calculated by the sum of all ratio values of each relevant question by the following formula and questions are rearranged from low to high order value/priority value [21]

$P_{o}=\sum_{j=1}^{k}$ all ratio values for $i^{\text {th }}$ relevant question

Step 3.5: Next, the affinity diagram [22] as shown in "Fig 2" is used to structure the customer voice or requirements ('WHATs'). The relevant questions are categorized in the different sections/groups named in the affinity diagram. The main group names in the affinity diagram are defined under the voice of customer section in the house of quality diagram and their Row weight against each voice of the customer is calculated by averaging the precedence or order value of the relevant questions in the sub groups.

Step 3.6: Technical requirements ('HOWs') are entered into the matrix and are arranged against ISO 9126 standards.

Step 3.7: The interrelationship matrix ('WHATs' vs. 'HOWs') is built to establish the connection between the voice of the customers and the technical design responses to improve the product. It is necessary to assign the relationship between them. If there is no relationship defined between them they are left blank otherwise a type of relation is assigned using different shape objects as shown in the "Fig 3".

Step 3.8: Next, the technical correlation matrix ('HOWs' vs. 'HOWs'), called roof of the House, is designed to identify where the different design attributes must work together else they will be in a design conflict. Here, in the house of quality, technical correlation is assigned in consultation with expert experience between design attributes in the form of positive $(+v e)$ and negative $(-v e)$ correlationship. 



Fig 2: Affinity diagram

Step 3.9: Next the column weight $(C W)$ is calculated by using the following formula:

$C W=\sum$ relationship weight $*$ row weight

The column weight represents the importance of the weight of the technical design attribute and ranked accordingly. It is observed that the design attributes with the highest column weights are the key attributes of the website(s) under observation that will contribute maximum to quality improvement.

Step 3.10: $\quad$ Finally, the designs targets are determined. The value written in the design target field is normalized in the range of $0-1$. If the goals(design atrributes) are achieved atleast by $0.8 \%$, it is considered as 'benchmarked' and 'achievable' goals and aims.

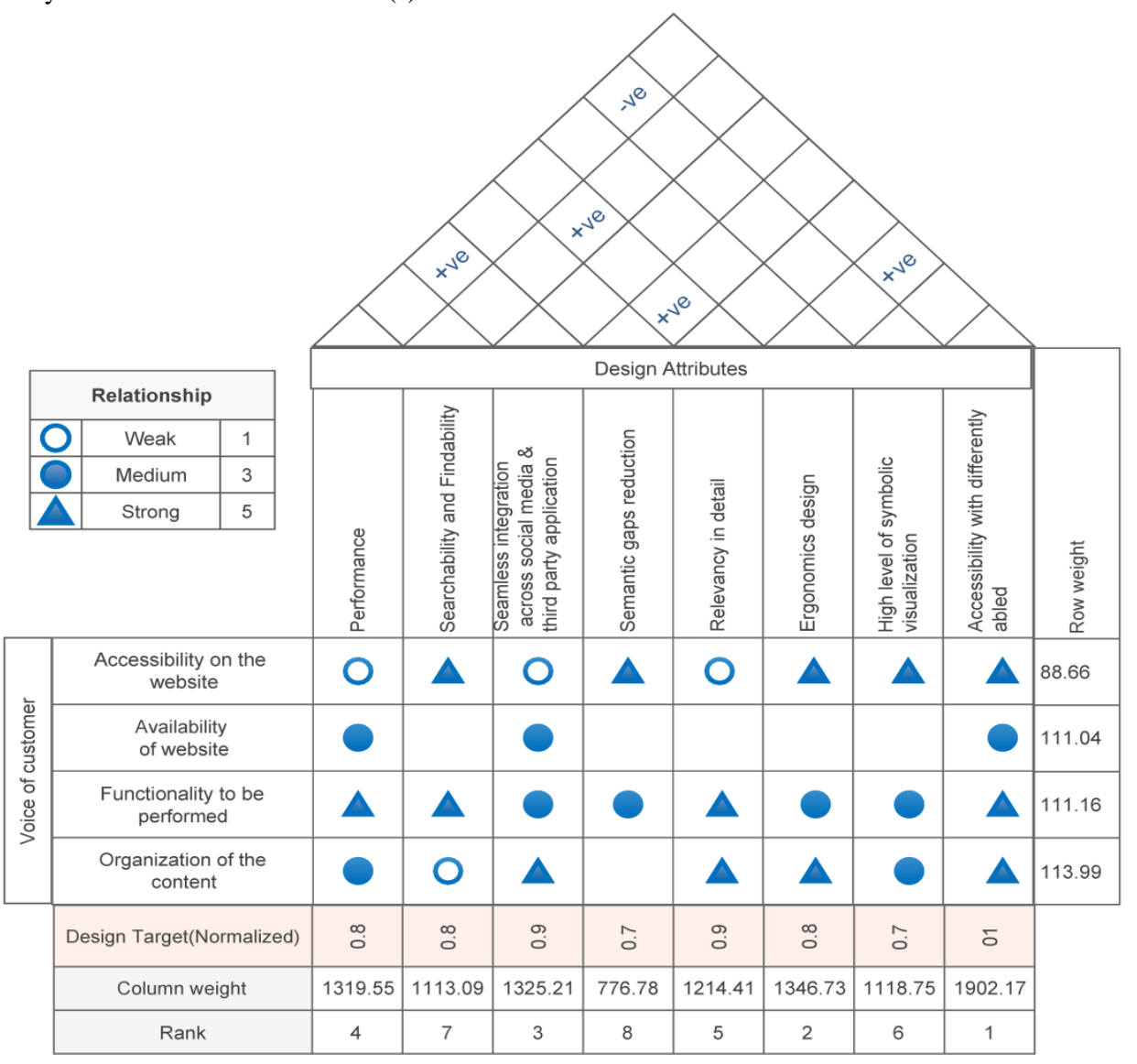

Fig 3: QFD for website under observation for Automatic Narration Generation System 
It is observed from the "Fig 3" that the design attributes are mapped with 79 relevant questions. The accessibility, design, integration with other social websites and performance are the key characteristics of the website that can contribute maximum to the website analysis process. These technical attributes have maximum weights that represent the work on these attributes bring the website to the highest level because it is the most important to resolve the issues related to these attributes that hinder the development of the website. The technical correlation matrix also helps in understanding the issues which are of greater importance from the view point of the technical team, because technical responses or design attributes are the mapping of the customer needs into the design attributes on the basis of ISO 9126 criteria.

Since the primary focus of this research work is to develop an automatic narration from the statistical data, it can be inferred that the customer pays more attention to the inadequacies related to more visual features of the website rather than mathematical analysis of various aspects of reporting. Therefore, for building a decision tree for automatic narration generation the authors will have some limitations in terms of having reporting section related to visual and aesthetic aspects of the website but statistically if navigation related statistics and rate related values are performing well it can be inferred that website has great visual content.

Step 4: The output narration generation algorithm, Deterministic Linear decision Tree (DLDT) is applied to the rearranged order of expectations of client's reporting need to generate a narration and to analyse the statistical data.

Step 5: After applying the above said algorithm, the values of the nodes of the tree are collaborated with the text explaining the impact of different values of statistical data to generate narration and are stored in the database again.

Step 6: The next module is the automated narration output based on above said algorithm i.e. DLDT to show narration on the reporting console. The output may be a html file, pdf, a word document etc.

\subsection{Main advantages}

- The implemented system generates a report which has the higher degree of accuracy and efficiency as structuring algorithm is sign based i.e. positive, negative or 0 .

- Ground truth validation and quality checks are performed by multiple reviewers using the Delphi techniques by having a substantial agreement between the raters.

- The system has the description and mathematical explanation of the events/actions narrated by the auto narrator.

- The system has the proper interpretation of the visual data along with storyboard.

- The objective evaluation of the narration is supported by the expertise's subjective agreement and validated against ground truth.

\section{LIMITATIONS}

- The Delphi technique is a time consuming process.

- There is a small possibility of disagreement between the experts on multiple aspects.
- There is a need for developing text analytics systems to collect the data like lexical density and readability index of a page etc. for the website.

- Only, the user requirements are gathered by using QFD but it may not be possible to incorporate all the requirements for the automatic narration generation as the report is statistical data based.

\section{DISCUSSION}

The research was carried out in automatic report writing of web analytics because the limited work has been reported in this context. The research hypothesis was that an algorithm can be built to write automatic narration of mobile and web analytics. The process of investigating the behavior or performance of the website on the computer's browser is called web analytics and on mobile device's browser is called mobile analytics. In the process of building a narrator that attempts to validate the hypothesis of automatic narration generation, it was found that evaluation of statistical data and performance parameters by the domain experts is of great importance and cannot be ignored. The report should have current statistical information on web analytics etc. and the expected output should be meaningful and technically sound. The report produced by the implemented system fulfills all the requirements. So in this research work the authors have brought accessibility with differently abled, Ergonomics design and integration across social media and third party applications etc. performance parameters ahead using QFD that can contribute the maximum to the website as these aspects of the reports were ignored by the other researchers. The ground truth validation and quality check aspects were also ignored. But this system took these aspects as a part by implementing the Delphi technique. The different 93 questions related to the website were reviewed by the 45 raters on the scale of 5 . The raters have a substantial agreement between them which was calculated by using Fleiss kappa formula i.e. $K=0.6210$ in the research work. The statistical data is further fed as the Deterministic linear decision tree algorithm is used for deciding the structure or plot of the narration. Most of the researchers took artificial intelligence based algorithms which generally are based on the classification of datasets and have a mathematical evaluation of the narration's events.

\section{RESULTS}

In this section, the outcomes of the implemented automatic narration generation process done are presented to validate results against Ground truth. These results try to evaluate and validate as per the objectives mentioned earlier and also try to address the validity of hypothesis taken for conducting this research. The system is accessible at www.paramjot.in.There are 4 possible outcomes of the research work on the basis of quality and appropriateness of 'reports' generated containing automatically generated narration. If the authors have to evaluate all these four outcomes they first need to conduct an exercise would design the experiments to cover all these four outcomes.

As per Design of Experiments full factorial method, the authors have one parameter and four possible outcomes for evaluation as shown in "Table 3" and calculated Precision and Recall values of generated Reports as shown in "Table 4". 
Table 3. Possible outcomes

\begin{tabular}{|l|l|l|}
\hline Parameter & $\begin{array}{l}\text { Description } \\
\text { of parameter }\end{array}$ & \multicolumn{1}{|c|}{ Description of outcomes } \\
\hline \multirow{3}{*}{ Appropriate } & $\begin{array}{l}\text { This means } \\
\text { the human } \\
\text { raters } \\
\text { considered the }\end{array}$ & $\begin{array}{l}\text { Auto narration got correctly } \\
\text { selected as appropriate. } \\
\text { Auto narration got correctly } \\
\text { rejected as inappropriate. }\end{array}$ \\
& $\begin{array}{l}\text { Auto narration got } \\
\text { automatically } \\
\text { generated } \\
\text { narration as } \\
\text { appropriate. }\end{array}$ & $\begin{array}{l}\text { appropriate. selected as } \\
\text { Auto narration got } \\
\text { incorrectly rejected. }\end{array}$ \\
\hline
\end{tabular}

Table 4. Precision and Recall

\begin{tabular}{|c|c|c|c|}
\hline $\begin{array}{c}\text { No. of } \\
\text { reports } \\
\text { in a set }\end{array}$ & $\begin{array}{c}\text { No. of selected } \\
\text { reports in a set }\end{array}$ & $\begin{array}{c}\text { Precision } \\
(\boldsymbol{P})\end{array}$ & $\begin{array}{c}\text { Recall } \\
(\boldsymbol{R})\end{array}$ \\
\hline$N_{s}$ & $R_{S}$ & $P=\frac{R_{s}}{N_{s}}$ & $R=\frac{R_{s}}{T_{r}}$ \\
\hline 2 & 2 & 1 & 0.09 \\
\hline 4 & 3 & 0.75 & 0.14 \\
\hline 3 & 2 & 0.67 & 0.09 \\
\hline 4 & 3 & 0.75 & 0.14 \\
\hline 3 & 2 & 0.67 & 0.09 \\
\hline 5 & 4 & 0.8 & 0.19 \\
\hline
\end{tabular}

Here total number of reports generated $\left(T_{r}\right)=21$.

Precision $(P)$ : the rate of a number of reports as selected appropriate $\left(R_{S}\right)$ with respect to a number of reports $\left(N_{S}\right)$ from a set of reports at ' $\mathrm{K}$ ' thresholds.

$\operatorname{Recall}(R)$ : the ratio of reports selected as appropriate $\left(R_{S}\right)$ with respect to the total number of reports generated $\left(T_{r}\right)$.

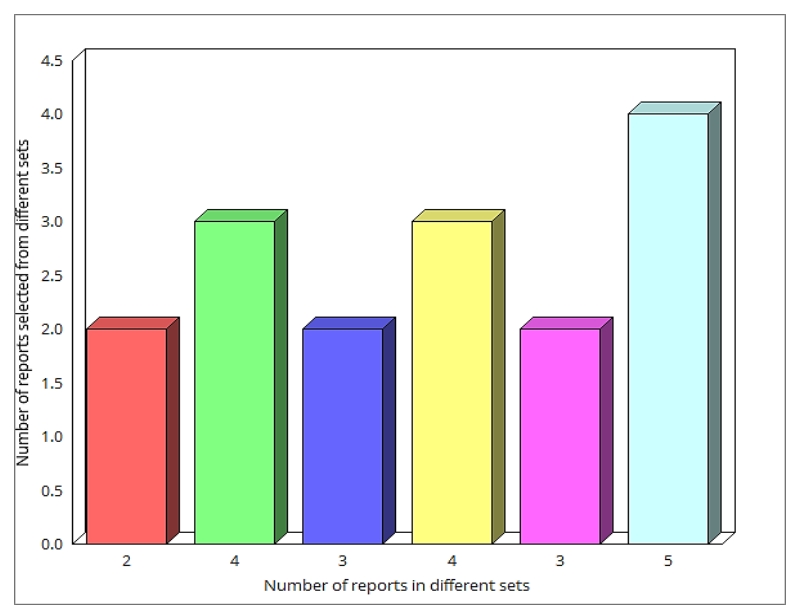

Fig 4: Graph representing number of reports selected from different sets

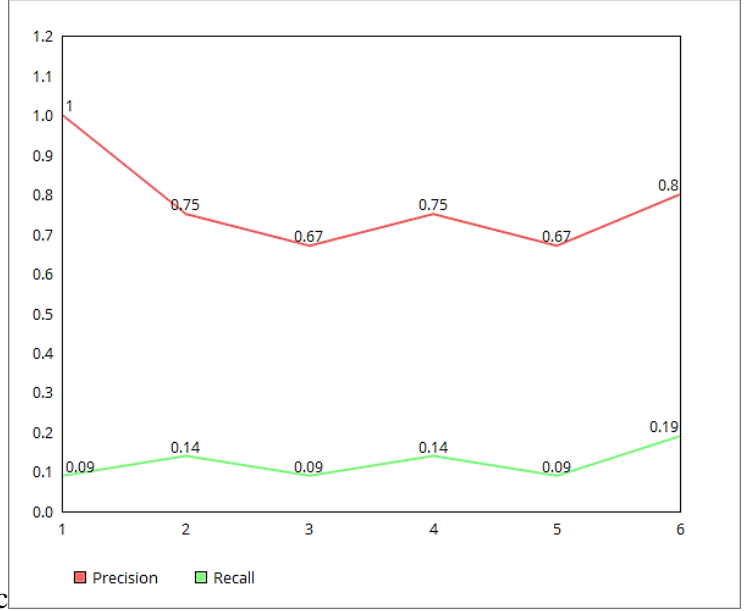

Fig 5: Graph representing precision and recall values of selected reports

It is clear from "Fig 5" that higher the value of the precision greater number of reports that are fulfilling the expectations of different experts. The higher value of precision also indicates that the quality of reports generated is also according to the expectation of the expert's. The different values of precisions are calculated by dividing the total number of reports into different sets by using random sampling. For example, in the research work, a number of sets are 6 and they contain a different number of reports say 2, 4, 3, 4, 3 and 5 as shown in above "Table 4". The values of precision depend upon the number of reports selected as appropriate from each set that are relevant at ' $\mathrm{K}$ ' threshold values. Here ' $\mathrm{K}$ ' has different values according to the size of the different sets. For example, Precision at 4 means $3 / 4$ for the second set. ' $K$ ' represents the number of reports in a set. It also indicates the number of relevant reports in $\mathrm{K}$ reports. Also, the higher value of recall indicates that the greater number of reports generated are complete in themselves and considered as appropriate with respect to a total number of reports generated. The sample report generated by Delphi based Narrator-Automatic narration generation system is shown in the "Fig 6". The Full report for the website www.punjabupdate.com is available at www.paramjot.in/punjab and for the website www.ijorcs.org is available at www.paramjot.in/ijorcs. 


\begin{tabular}{|c|c|c|c|}
\hline \multicolumn{4}{|c|}{$\begin{array}{c}\text { Automatic Narration Generation } \\
\text { punjabupdate.com website data } \\
\text { Week of May.07 - May.14,2016 }\end{array}$} \\
\hline Metric & Current week value & Rate of change & \\
\hline Primary Content Consumption & 0.34 & $0 \%$ change from last week & \\
\hline Repeat Visitor Ratio & $10.64 \%$ & $-27.86 \%$ ' 1 from last week & \\
\hline \multicolumn{4}{|c|}{ Event Conversion Rate } \\
\hline Sessions with Events & 2732 sessions & $0.81 \%$ B from last week & \\
\hline Conversion Rate & $23.89 \%$ & $-10.19 \%$ 'P from last week & \\
\hline Bounce Rate & $7.22 \%$ & $-1.03 \%$ is from last week & \\
\hline \multicolumn{4}{|c|}{ Basic Metrics } \\
\hline Sessions & 32456 sessions & $31.23 \%$ 1 from last week & \\
\hline Users & 31336 users & $32.05 \%$ 1 from last week & \\
\hline Pageviews & 149287 pageviews & $31.6 \%$ B from last week & \\
\hline
\end{tabular}

Fig 6: Partial report generated by Automatic Narration Generation System

\section{CONCLUSION}

Based on this research work, it can be safely concluded that there is still need for building an automatic narration generation systems that are based on the ground truth validation. Multiple approaches were reviewed and based on the identified challenges and gaps, steps of this research work were implemented. The main contribution is the development of new framework for creating an automatic narration of analytics of websites and mobile apps. This has been done using the 'Delphi technique' to build narration content tree or content format. QFD was used to understand the user requirements and expected features of auto narration system. Experts were consulted with the help of a well-structured questionnaire and a substantial amount of agreement i.e. $K=0.6210$ has been calculated between the experts for final decision in making narration tree content. Now, with the help of calculations related to the rate of change (Positive, Negative, No Change or abnormal change) the narration content is extracted from the Deterministic Linear Decision Tree Structure. Last, but not the least, this narration is evaluated for ground truth validation with the help of experts dealing with data analytics recall/precision values. For the future direction, it is suggested that more systems may be developed that are Delphi based in the other domains like political elections commentary, weather forecasting, data journalism etc. The system can also be developed further by the usage of deep learning algorithms and neural networks for auto generating the narration. The future work may be aligned to overcome the limitations mentioned in the paper.

\section{REFERENCES}

[1] Narrative Science. (2016, April) narrativescience.com. [Online]. https://www.narrativescience.com/quill-engage

[2] Adobe. (2016, April) adobe.com. [Online]. http://www.adobe.com/in/marketing-cloud/webanalytics.html

[3] Stephen W. Littlejohn and Karen A. Foss, Theories of Human Communication, 10th ed. Long Grove, Illinois, USA: Waveland Press, 2010.

[4] Charlie Hargood, David E Millard, and Mark J Weal, "Investigating a thematic approach to narrative," in In
DAH 09 at Hypertext 09, 20th ACM conference on Hyperetext and Hypermedia, Torino, Italy, 2009.

[5] R. Michael Young, "Story and discourse: A bipartite model of narrative generation in virtual worlds," in Interaction Studies: Social Behaviour and Communication in Biological and Artificial Systems, vol. 8, no. 2, pp. 177-208, 2006.

[6] Charles B. Callaway and James C. Lester, "Narrative Prose Generation," Artificial Intelligence, vol. 139, no. 2, pp. 213-252, August 2002.

[7] Pablo Gervás and Carlos León, "The Need for MultiAspectual Representation of Narratives in Modelling their Creative Process," in In Proceedings of International Workshop on Computational Models of Narrative, Quebec City, Canada, 2014, pp. 1-16.

[8] Takashi Ogata, Satoki Umehara, Sayaka Yamakage, Koji Ueda, and Yoshinori Hosaka, "Aspects of Narrative Discourse Process and Their Integration by Computer Simulation," University of Yamanashi, Japan, Takeda Kofu, Japan, technical report 2004.

[9] Craig A. Lindley, "Story and Narrative Structures in Computer Games", Brunhild Bushoff, Ed. Munich, Germany: High Text Verlag, 2005.

[10] Albert Gatt and Francois Portet, "Text content and task performance in the evaluation of a Natural Language Generation system," in In Proceedings of the International Conference on Recent Advances in Natural Language Processing, Borovets, Bulgaria, 2009, pp. 107112.

[11] Google. Google Analytics - Mobile, Premium and Free Website Analytics - Google. [Online]. https://www.google.co.in/analytics

[12] Google. Google Analytics Google Developers. [Online]. https://developers.google.com/analytics

[13] Paramjot Kaur Sarao, Puneet Mittal, and Gursharan Singh Dhanjal. (2016) Questions. [Online]. http://paramjot.in/frontpages/questions 
[14] Paramjot Kaur Sarao, Puneet Mittal, and Gursharan Singh Dhanjal. (2016) Scales. [Online]. http://paramjot.in/frontpages/scales

[15] Paramjot Kaur Sarao, Puneet Mittal, and Gursharan Singh Dhanjal. (2016) Responses. [Online]. http://paramjot.in/frontpages/responses

[16] Chia-Chien Hsu and Brian A. Sandford, "The Delphi Technique: Making Sense Of Consensus," Practical Assessment, Research \& Evaluation, vol. 12, no. 10, pp. 1-8, 2007.

[17] Wikipediacontributors. (2016, January) en.wikipedia.org. [Online]. https://en.wikipedia.org/wiki/Fleiss\%27_kappa

[18] Mamoon O.H. Saeed, "Web Development using Quality Function Deployment," University of Leeds, UK, Msc. information systems 2004
[19] Paramjot Kaur Sarao, Puneet Mittal, and Gursharan Singh Dhanjal. (2016) Priority. [Online]. http://paramjot.in/frontpages/precedence

[20] Paramjot Kaur Sarao, Puneet Mittal, and Gursharan Singh Dhanjal. (2016) Priority of relevant questions. [Online]. http://paramjot.in/frontpages/relevantquestionsprecedenc e

[21] Paramjot Kaur Sarao, Puneet Mittal, and Gursharan Singh Dhanjal. (2016) Final questions. [Online] http://paramjot.in/frontpages/finalquestions

[22] Hugh Beyer and Karen Holtzblatt, Contextual Design: Defining Customer-centered Systems. San Francisco, USA: Morgan Kaufmann, 1998, 1998. 